\title{
Splenic Small Lymphocytic Lymphoma
}

National Cancer Institute

\section{Source}

National Cancer Institute. Splenic Small Lymphocytic Lymphoma. NCI Thesaurus. Code C7304.

Small lymphocytic lymphoma occurring in the spleen. 